\title{
Diagnosis and outcomes of acute kidney injury using surrogate and imputation methods for missing preadmission creatinine values
}

Amélie Bernier-Jean ${ }^{1,2}$, William Beaubien-Souligny ${ }^{1,2}$, Rémi Goupil ${ }^{1,2}$, François Madore ${ }^{1,2}$, François Paquette ${ }^{1,2}$, Stéphan Troyanov ${ }^{1,2}$ and Josée Bouchard ${ }^{1,2^{*}}$ (D)

\begin{abstract}
Background: Missing preadmission serum creatinine ( $\mathrm{SCr}$ ) values are a common obstacle to assess acute kidney injury (AKI) diagnosis and outcomes. The Kidney Disease Improving Global Outcomes (KDIGO) guidelines suggest using a SCr computed from the Modification of Diet in Renal Disease (MDRD) with an estimated glomerular filtration rate of $75 \mathrm{ml} / \mathrm{min} / 1.73 \mathrm{~m}^{2}$. We aimed to identify the best surrogate method for baseline $\mathrm{SCr}$ to assess AKI diagnosis and outcomes.

Methods: We compared the use of 1) first SCr at hospital admission 2) minimal SCr over 2 weeks after intensive care unit admission 3) MDRD computed SCr and 4) Chronic Kidney Disease Epidemiology Collaboration (CKD-EPI) computed $\mathrm{SCr}$ to assess AKI diagnosis and outcomes. We then performed multilinear regression models to predict preadmission $\mathrm{SCr}$ and imputation strategies to assess AKI diagnosis.

Results: Our one-year retrospective cohort study included 1001 critically ill adults; 498 of them had preadmission SCr values. In these patients, AKI incidence was $25.1 \%$ using preadmission SCr. First SCr had the best agreement for AKI diagnosis (22.5\%; kappa $=0.90$ ) and staging (kappa $=0.81)$. MDRD, CKD-EPI and minimal SCr overestimated AKI diagnosis (26.7\%, 27.1\% and 43.2\%;kappa =0.86, 0.86 and 0.60, respectively). However, MDRD and CKD-EPI computed SCr had a better sensitivity than first SCr for AKI ( $93 \%$ and $94 \%$ vs. 87\%). Eighty-eight percent of patients experienced renal recovery at least 3 months after hospital discharge. All methods except the first SCr significantly underestimated the percentage of renal recovery. In a multivariate model, age, male gender, hypertension, heart failure, undergoing surgery and log first SCr best predicted preadmission SCr (adjusted $\mathrm{R}^{2}=0.56$ ). Imputation methods with first $\mathrm{SCr}$ increased AKI incidence to 23.9\% (kappa = 0.92) but not with MDRD computed SCr (26.7\%;kappa $=0.89$ ).

Conclusion: In our cohort, first SCr performed better for AKI diagnosis and staging, as well as for renal recovery after hospital discharge than MDRD, CKD-EPI or minimal SCr. However, MDRD SCr and CKD-EPI SCr improved AKI diagnosis sensitivity. Imputation methods minimally increased agreement for AKI diagnosis.
\end{abstract}

Keywords: Acute kidney injury, Baseline creatinine, Diagnosis, Epidemiology, Surrogate, Outcomes

\footnotetext{
*Correspondence: josee.bouchard.1@umontreal.ca

'Department of Medicine, Division of Nephrology, Sacre-Coeur Hospital of

Montreal, 5400 Gouin Blvd West, Montreal, Quebec H4J 1C5, Canada

${ }^{2}$ Faculty of Medicine, University of Montreal, Montreal, Quebec, Canada
} 


\section{Background}

Acute kidney injury (AKI) is associated with higher mortality, longer hospital stays, and a higher likelihood of developing chronic kidney disease (CKD) [1-3]. AKI diagnosis relies on the quantification of changes in serum creatinine (SCr) from a preadmission value [4]. However, preadmission $\mathrm{SCr}$ is missing in $25-50 \%$ of patients [5-7], thereby being a major obstacle to accurately assess AKI diagnosis and outcomes, as recently highlighted by Siew and colleagues [8].

When baseline serum creatinine is missing, the Acute Dialysis Quality Initiative and Kidney Disease Improving Global Outcomes (KDIGO) guidelines suggest using a baseline SCr computed from the Modification of Diet in Renal Disease (MDRD) formula, assuming an estimated glomerular filtration rate (eGFR) of $75 \mathrm{ml} / \mathrm{min}$ per $1.73 \mathrm{~m}^{2}[4,9]$. This "ad hoc" suggestion was not a formal recommendation, due to the limited evidence on this issue. Some studies have assessed the performance of the MDRD or other surrogate methods for missing preadmission SCr for AKI diagnosis [5, 7, 10-17]. However, several factors other than age, race and gender may influence SCr levels, like comorbidities, fluid balance and prolonged hospital stay [18-20]. To our knowledge, only one study has attempted to improve the predictive performance of surrogate methods by including clinical characteristics into predictive models [12]. In that study, the full imputation method required variables not readily available, which may hinder its widespread use in clinical research.

In this study, our objectives were to 1) identify the most accurate surrogate method among those commonly used to estimate baseline SCr for AKI diagnosis and outcomes 2) identify variables associated with AKI diagnosis misclassification and 3) determine the value of imputation strategies to improve the capacity of estimating AKI diagnosis beyond surrogate methods.

\section{Methods}

\section{Study design and participants}

We performed a retrospective study of critically ill adult patients admitted to our tertiary care academic center between January $1^{\text {st }}$ and December $31^{\text {st }}, 2012$. In this study, we assessed the performance of four surrogate methods: 1) first SCr level at hospital admission; 2) minimal SCr level within 2 weeks after intensive care unit (ICU) admission; 3) SCr computed from the MDRD formula [21] for an eGFR of $75 \mathrm{ml} / \mathrm{min}$ per $1.73 \mathrm{~m}^{2}[4,9]$ and 4) SCr computed from the Chronic Kidney Disease Epidemiology Collaboration (CKD-EPI) formula [22] for an eGFR of $75 \mathrm{ml} / \mathrm{min}$ per $1.73 \mathrm{~m}^{2}$. We performed a multilinear regression model to identify patients characteristics that best predict preadmission SCr. We then performed imputation strategies using calculated $\mathrm{SCr}$ values from the multilinear regression models to assess AKI diagnosis.

We included randomly selected critically ill patients aged 18 or more, and excluded readmissions, patients on chronic dialysis, those having a kidney transplant, or who stayed in the ICU less than $24 \mathrm{~h}$. We followed the STrengthening the Reporting of OBservational studies in Epidemiology (STROBE) guidelines for observational studies [23].

\section{Data collection}

We collected data on demographic characteristics, past medical history, laboratory results, severity of illness and processes of care from our ICU electronic medical record and by chart review. We retrieved $\mathrm{SCr}$ levels for each patient for up to one year before hospital admission. Outcomes included AKI diagnosis and staging, mortality at hospital discharge, and renal recovery at least 3 months after hospital discharge.

\section{Definitions}

We used the KDIGO SCr criteria for AKI diagnosis and staging [4]; urine output criteria were not considered. AKI could be diagnosed if 1) there was an increase in serum creatinine $(\mathrm{SCr})$ by $\geq 26.5 \mathrm{umol} / \mathrm{l}(\geq 0.3 \mathrm{mg} / \mathrm{dl})$ within $48 \mathrm{~h}$ or 2 ) an increase in $\mathrm{SCr}$ to $\geq 1.5$ times baseline, which is known or presumed to have occurred within the prior 7 days. We included creatinine values up to $48 \mathrm{~h}$ before ICU admission to allow for AKI assessment according to the first criterion. By definition, the 48 - $\mathrm{h}$ criterion does not require a baseline creatinine value. We defined renal recovery by a decrease in $\mathrm{SCr}$ within $150 \%$ of baseline SCr. Preadmission or baseline $\mathrm{SCr}$ was defined as the closest value between 3 and 12 months before hospital admission to reflect CKD status [24], and if unavailable, the furthest value between 3 months before hospital admission and hospital admission. Preadmission SCr was considered missing if no $\mathrm{SCr}$ values were available before admission. Baseline eGFR was computed with MDRD using preadmission $\mathrm{SCr}$, and CKD status was defined as an eGFR less than $60 \mathrm{ml} / \mathrm{min}$ per $1.73 \mathrm{~m}^{2}$ [24]. We also defined CKD status by examining medical records. Sequential Organ Failure Assessment (SOFA) scores were assessed at ICU admission [25]. Cumulative fluid balance was the sum of daily fluid balances during the first week of ICU admission.

\section{Statistical analyses and sample size calculation}

Continuous variables are presented as mean \pm standard deviation or median and interquartile range (IQR) and compared using $t$-test or Mann-Whitney $\mathrm{U}$ test, where appropriate. Categorical variables are presented as proportions and compared using $x^{2}$ test. We compared preadmission $\mathrm{SCr}$ and each surrogate method with the 
Bland-Altman method. For AKI diagnosis, we reported the different sensitivity and specificity for each surrogate method compared to preadmission $\mathrm{SCr}$, and used the kappa statistic with $95 \%$ confidence intervals to report the level of agreement between the different surrogate methods and preadmission SCr. Misclassification rates were calculated as the proportion of patients incorrectly classified as AKI or non-AKI based on preadmission SCr, and we compared well-classified and misclassified AKI using the McNemar test. We reported similar statistics for AKI staging. We performed a multilinear regression analysis to predict preadmission $\mathrm{SCr}$ using variables associated with AKI misclassification and surrogate methods that had good predictive performance for AKI diagnosis. We used log-transformed creatinine for all $\mathrm{SCr}$ values. We then imputed the predicted $\mathrm{SCr}$ values from the different multilinear regression models (imputation strategy) to assess their effect on the incidence of AKI.

Statistical tests were two-sided and $p$ values were reported. Appropriate adjustments for the Bonferroni correction were mentioned in tables with multiple $p$ value comparisons. Statistical analyses were performed with SPSS, version 20.0 (IBM, Armonk, NY) and SAS 9.3 (SAS Institute, Cary, NC).

A sample size of 1073 subjects would achieve a 80\% study power at a type 1 error of 0.05 to detect an absolute $5 \%$ difference in the incidence of AKI.

\section{Results}

Among 2464 patients admitted over a year, 1073 (43.\%) were randomly selected for the study population. From these, we excluded 17 patients (1.6\%) who were readmitted over the same period, 17 patients on chronic hemodialysis (1.6\%), one kidney transplant recipient $(0.1 \%)$, and 37 patients who stayed in the ICU less than $24 \mathrm{~h}(3.4 \%)$ (Fig. 1). Among the remaining 1001 patients, 498 (50\%) had a preadmission SCr value available and 121 (12\%) suffered from CKD. Preadmission SCr were measured between 3 to 12 months prior to admission in 341 patients $(68 \%)$, between 7 days to 3 months in 138 (28\%), and less than 7 days prior to admission for 19 patients (4\%).

\section{Characteristics of patients with and without preadmission creatinine}

Table 1 included the demographic, comorbidities, and processes of care of the study population with and without available preadmission SCr. Out of 1001 patients, almost all were from Caucasian or Asian origin (97.1\%, $n=972), 60.5 \%(n=606)$ were male, and median age was 66 (IQR 56-75) years old. Patients with missing preadmission $\mathrm{SCr}$ were younger, less likely to suffer from chronic obstructive pulmonary disease (COPD), heart failure, cirrhosis, diabetes, CKD, cancer, underwent less surgical procedures but more often required mechanical

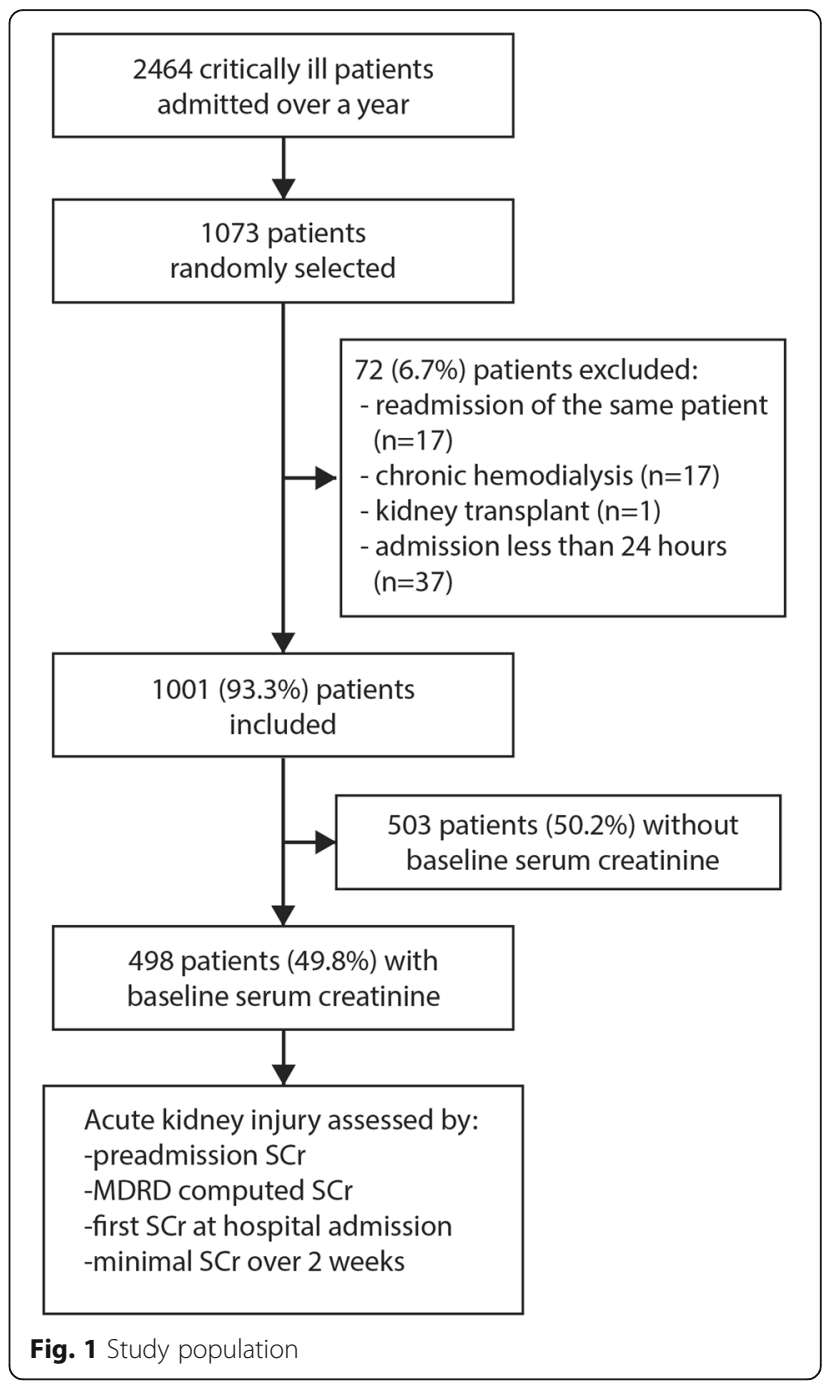

ventilation. The severity of illness score, use of vasopressors and acute renal replacement therapy (RRT) were similar between groups, as well as lengths of stay (LOS) and mortality rates.

\section{Acute kidney injury diagnosis $(n=498)$}

The AKI incidence according to preadmission $\mathrm{SCr}$ was 25.1\% (95\% CI 21.5-29.1\%). The first SCr method had the best agreement for AKI diagnosis (incidence of $22.5 \%$, kappa $=0.90)($ Table 2) but showed significant AKI misclassification with preadmission $\mathrm{SCr} \quad(p<0.004)$. Minimal SCr, MDRD and CKD-EPI computed SCr all overestimated AKI incidence (43.2\%, 26.7\%, 27.1\%; kappa scores $=0.60,0.86$, and 0.86, respectively). Minimal SCr showed a significant misclassification rate $(p<0.0001)$ but not MDRD or CKD-EPI computed SCr methods. The MDRD and CKD-EPI methods were associated with a better sensitivity for AKI diagnosis than the first SCr $(92.8 \%$ and $93.6 \%$ vs. $87.2 \%$ ), at the expense of a slightly lower specificity ( $95.4 \%$ and $95.1 \%$ vs. $99.2 \%)$. We also compared 
Table 1 Baseline characteristics of patients

\begin{tabular}{|c|c|c|c|}
\hline & With preadmission creatinine $(n=498)$ & Without preadmission creatinine $(n=503)$ & $p$ \\
\hline $\begin{array}{l}\text { Age (years) } \\
\text { Gender (male \%) } \\
\text { Race (\%) }\end{array}$ & $\begin{array}{l}67(58-76) \\
59.4\end{array}$ & $\begin{array}{l}65(53-75) \\
61.6\end{array}$ & $\begin{array}{l}0.005 \\
0.48\end{array}$ \\
\hline $\begin{array}{l}\text { Caucasian/Asian } \\
\text { Body mass index } \\
\text { Known preadmission creatinine }(\mu \mathrm{mol} / \mathrm{l})\end{array}$ & $\begin{array}{l}96.4 \\
27(24-32)(n=251) \\
73(60-90)\end{array}$ & $\begin{array}{l}97.8 \\
27(24-31)(n=261) \\
n / a\end{array}$ & $\begin{array}{l}0.18 \\
0.63\end{array}$ \\
\hline Baseline GFR MDRD (ml/min/1.73 $\left.\mathrm{m}^{2}\right)$ & $89(71-111)$ & $\mathrm{n} / \mathrm{a}$ & \\
\hline $\begin{array}{l}\text { CAD (\%) } \\
\text { COPD (\%) } \\
\text { Heart failure (\%) } \\
\text { Cirrhosis (\%) } \\
\text { Diabetes (\%) } \\
\text { Hypertension (\%) } \\
\text { CKD by history (\%) } \\
\text { CKD MDRD }<60 \mathrm{ml} / \mathrm{min} / 1.73 \mathrm{~m}^{2} \\
\text { Cancer } \\
\text { Surgery (\%) } \\
\text { SOFA score non-renal } \\
\text { SOFA score } \\
\text { Mechanical ventilation (\%) }\end{array}$ & $\begin{array}{l}36.7 \\
22.5 \\
12.9 \\
3.2 \\
29.1 \\
60.8 \\
14.9 \\
15.7 \\
29.5 \\
51.6 \\
4(2-7) \\
5(2-8) \\
37.1\end{array}$ & $\begin{array}{l}39.4 \\
12.3 \\
7.0 \\
1.0 \\
22.9 \\
55.7 \\
9.3 \\
\text { n/a } \\
12.9 \\
35.2 \\
5(2-8) \\
5(2-8) \\
48.3\end{array}$ & $\begin{array}{l}0.39 \\
<0.001 \\
0.002 \\
0.02 \\
0.02 \\
0.10 \\
0.007 \\
<0.001 \\
<0.001 \\
0.08 \\
0.21 \\
<0.001\end{array}$ \\
\hline Vasopressors (\%) & 27.9 & 33.0 & 0.08 \\
\hline Cumulative fluid balance $(\mathrm{L})$ & $0.9(-0.6-2.5)$ & $0.8(-0.7-2.4)$ & 0.42 \\
\hline RRT & 2.8 & 3.8 & 0.39 \\
\hline $\begin{array}{l}\text { Hospital LOS } \\
\text { Mortality }\end{array}$ & $\begin{array}{l}10(6-21) \\
12.9\end{array}$ & $\begin{array}{l}10(6-23) \\
11.7\end{array}$ & $\begin{array}{l}0.77 \\
0.59\end{array}$ \\
\hline
\end{tabular}

CAD coronary artery disease, COPD chronic obstructive pulmonary disease, CKD chronic kidney disease, SOFA Sequential Organ Failure Assessment Score, RRT renal replacement therapy, LOS length of stay

Data were missing in $<1 \%$ of records

preadmission $\mathrm{SCr}$ and each surrogate method with the Bland-Altman method (Additional file 1: Figures S1a-S1d).

\section{Acute kidney injury staging $(n=498)$}

The first $\mathrm{SCr}$ was the most accurate method for AKI staging, with only 37 misclassified patients (7.4\%) (Table 3). In comparison, using minimal SCr, 130 patients (26.1\%) were misclassified (Table 4), with MDRD computed SCr, 61 patients (12.2\%) (Table 5), and with CKD-EPI, 65 patients (13.1\%) (Table 6). Thirteen patients required acute dialysis for AKI and were therefore classified as AKI stage 3, irrespective of $\mathrm{SCr}$ values, as defined by the KDIGO criteria [4]. When considering only moderate to severe AKI (stages 2 and 3) as AKI and stage 1 as non-AKI,

Table 2 Acute kidney injury agreement between surrogate methods for missing baseline creatinine and preadmission serum creatinine

\begin{tabular}{|c|c|c|c|c|c|}
\hline & $\begin{array}{l}\text { AKI (\%) } \\
n=498\end{array}$ & Sensitivity & Specificity & Kappa & McNemar* \\
\hline Preadmission creatinine & $\begin{array}{l}25.1 \\
(95 \% \text { Cl } 21.5-29.1 \% ; n=125)\end{array}$ & - & - & - & \\
\hline First serum creatinine at hospital admission & $\begin{array}{l}22.5 \\
(95 \% \text { Cl } 19.0-26.4 \% ; n=112)\end{array}$ & 87.2 & 99.2 & $\begin{array}{l}0.895 \\
(95 \% \text { Cl 0.848-0.942) }\end{array}$ & 0.004 \\
\hline Minimal creatinine within 2 weeks after ICU admission & $\begin{array}{l}43.2 \\
(95 \% \text { Cl 38.9-47.6\%; } n=215)\end{array}$ & 98.4 & 75.3 & $\begin{array}{l}0.595 \\
(95 \% \text { Cl } 0.526-0.664)\end{array}$ & $<0.0001$ \\
\hline MDRD computed serum creatinine & $\begin{array}{l}26.7 \\
(95 \% \text { Cl } 23.0-30.8 \% ; n=133)\end{array}$ & 92.8 & 95.4 & $\begin{array}{l}0.864 \\
(95 \% \text { Cl 0.813-0.915) }\end{array}$ & 0.169 \\
\hline CKD-EPI computed serum creatinine & $\begin{array}{l}27.1 \\
(95 \% \text { Cl } 23.4-31.2 ; n=135)\end{array}$ & 93.6 & 95.1 & $0.865(95 \%$ Cl 0.814-0.916) & 0.076 \\
\hline
\end{tabular}

${ }^{*} p<0.01$ represented significant difference, with the Bonferroni correction

Misclassification rates were $3.8 \%$ for the first $\mathrm{SCr}$ (overestimation $0.6 \%$ and underestimation $3.2 \%$ ), $18.9 \%$ for the minimal SCr (overestimation $18.5 \%$ and underestimation $0.4 \%$ ), 5.2\% with MDRD (overestimation 3.4\% and underestimation $1.8 \%$ ), 5.2\% with CKD-EPI (overestimation $3.6 \%$ and underestimation $1.6 \%$ ) MDRD: Modification of Diet in Renal Disease 
Table 3 Acute kidney injury staging according to first creatinine at hospital admission compared to preadmission creatinine

\begin{tabular}{lllllll}
\hline & & \multicolumn{6}{l}{ Preadmission serum creatinine } \\
\cline { 3 - 7 } & & No AKI & 1 & 2 & 3 & total \\
\hline First SCr & No AKI & 370 & 7 & 4 & 5 & 386 \\
& 1 & 3 & 64 & 10 & 1 & 78 \\
2 & 0 & 3 & 6 & 2 & 11 \\
& 3 & 0 & 0 & 2 & 21 & 23 \\
& Total & 373 & 74 & 22 & 29 & 498 \\
\hline
\end{tabular}

Kappa 0.811 (95\% Cl 0.756-0.866)

Misclassification rate was $7.4 \%$ (overestimation $1.6 \%$ and underestimation $5.8 \%)$ - McNemar $p=0.02$

$p<0.01$ represented significant difference, with the Bonferroni correction

the misclassification rates were $5.4 \%(n=27)$ for the first SCr, 9.8\% $(n=49)$ with minimal SCr, $7.8 \%(n=39)$ with MDRD SCr, and $8.4 \%(n=42)$ with CKD-EPI.

\section{Renal recovery and mortality rates}

The AKI in-hospital mortality rate using preadmission SCr was $25.6 \%(n=32 / 125 ; 95 \%$ CI $18.8-33.9 \%)$. There were no differences in AKI mortality rates between the different surrogate methods and preadmission SCr. The mortality rate with first $\mathrm{SCr}$ was $26.8 \%(n=30 / 112$; $95 \%$ CI $19.5-35.7 \% ; p=0.86)$, with minimal SCr, $20.9 \%(n=$ $45 / 215 ; 95 \%$ CI 16.0-26.9\%; $p=0.32)$, with MDRD computed SCr, 27.1\% ( $n=36 / 133$; 95\% CI 20.2-35.2\%; $p=0.79)$ and with CKD-EPI, 26.7\% $(n=36 / 135 ; 95 \%$ CI 19.9-34.7\%; $\mathrm{p}=0.84$ ).

After hospital discharge, according to preadmission SCr, $88.0 \%$ of AKI patients (95\% CI 78.7-93.6\%; $n=66$ / 75 , excluding $n=18$ with missing data) recovered their kidney function after at least 3 months. The first $\mathrm{SCr}$ was the only method associated with a similar percentage of renal recovery rate compared to preadmission $\mathrm{SCr}$ (89.4\% 95\% CI 79.7-94.8\%; $n=59 / 66$ excluding $n=16$ missing data; $p=0.79)$. All other methods were associated with a significantly lower percentage of renal recovery, namely minimal SCr (59.0\%;95\% CI 50.7-66.8\%; $n=82 / 139$ excluding $n=31$ missing data; $<0.001)$, MDRD

Table 4 Acute kidney injury staging according to minimal creatinine compared to preadmission creatinine

\begin{tabular}{lllllll}
\hline & & \multicolumn{5}{c}{ Preadmission serum creatinine } \\
\cline { 3 - 7 } & & No AKI & 1 & 2 & 3 & Total \\
\hline Minimal Scr & No AKI & 281 & 1 & 0 & 1 & 283 \\
& 1 & 80 & 52 & 7 & 3 & 142 \\
& 2 & 12 & 18 & 12 & 2 & 44 \\
3 & 0 & 3 & 3 & 23 & 29 \\
& Total & 373 & 74 & 22 & 29 & 498
\end{tabular}

Kappa 0.502 (95\% Cl 0.437-0.567)

Misclassification rate was $26.1 \%$ (overestimation $23.3 \%$ and underestimation $2.8 \%)$ - McNemar $p<0.0001$

$p<0.01$ represented significant difference, with the Bonferroni correction
Table 5 Acute kidney injury staging according to MDRD computed creatinine compared to preadmission creatinine

\begin{tabular}{lllllll}
\hline & \multicolumn{5}{l}{ Preadmission serum creatinine } \\
\cline { 3 - 7 } & No AKI & 1 & 2 & 3 & Total \\
\hline MDRD Scr & No AKI & 356 & 7 & 2 & 0 & 365 \\
& 1 & 15 & 47 & 7 & 1 & 70 \\
& 2 & 2 & 10 & 10 & 4 & 26 \\
& 3 & 0 & 10 & 3 & 24 & 37 \\
& Total & 373 & 74 & 22 & 29 & 498 \\
\hline
\end{tabular}

MDRD: Modification of Diet in Renal Disease

Kappa 0.711 (95\% Cl 0.650-0.771)

Misclassification rate was $12.2 \%$ (overestimation $8.0 \%$ and underestimation $4.2 \%)$ - McNemar $p=0.05$

$p<0.01$ represented significant difference, with the Bonferroni correction

(63.3\%;95\% CI 52.3-73.1\%; $n=50 / 79$ excluding $n=18$ missing data; $p<0.001)$ and CKD-EPI $(59.3 \%$;95\% CI 48.4$69.3 \% ; n=48 / 81$ excluding $n=18$ missing data; $p<0.001)$.

\section{Clinical characteristics associated with misclassified AKI}

We compared well-classified and misclassified AKI by the surrogate method with the highest level of agreement, i.e. the first SCr at hospital admission. Misclassified patients were more likely to be female, and to suffer from CKD. They had a higher SOFA score, used vasopressors more frequently, were less likely to suffer from hypertension or undergo surgery (Additional file 2: Table S1a). We also compared well-classified and misclassified AKI by MDRD computed SCr (Additional file 2: Table S1b). Misclassified patients were more likely to suffer from heart failure, diabetes, and CKD, and had a higher SOFA score, while they were less likely to suffer from COPD, cancer or undergo surgery. We further detailed misclassified AKI by missed and overdiagnosed AKI in Additional file 2: Table S1c and d.

We also assessed whether cumulative fluid balance and ICU length of stay were different between wellclassified and misclassified AKI using the minimal SCr, as positive fluid balance and longer ICU stay may affect

Table 6 Acute kidney injury staging according to CKD-EPI computed creatinine compared to preadmission creatinine

\begin{tabular}{lllllll}
\hline & \multicolumn{6}{l}{ Preadmission serum creatinine } \\
\cline { 3 - 7 } CKD-EPI Scr & No AKI & 1 & 2 & 3 & Total \\
\hline & 1 & 355 & 7 & 1 & 0 & 363 \\
& 2 & 2 & 46 & 8 & 1 & 71 \\
& 3 & 0 & 11 & 8 & 4 & 25 \\
& Total & 373 & 74 & 22 & 29 & 498 \\
\hline
\end{tabular}

CKD-EPI: Chronic Kidney Disease Epidemiology Collaboration Kappa 0.694 (95\% Cl 0.633-0.755)

Misclassification rate was $13.1 \%$ (overestimation $8.8 \%$ and underestimation $4.2 \%)$ - McNemar $p=0.04$

$p<0.01$ represented significant difference, with the Bonferroni correction 
subsequent $\mathrm{SCr}$ levels and therefore, AKI diagnosis [18-20]. Neither cumulative fluid balance nor ICU length of stay was significantly associated with AKI misclassification using minimal SCr.

\section{Multilinear regression model and imputation strategies}

We included in our multilinear regression model clinical characteristics that were associated with misclassified AKI rates (Additional file 2: Table S1a and S1b) and surrogate methods that had a good predictive capacity for AKI diagnosis (first SCr and MDRD computed SCr). We did not include vasopressors as they are part of SOFA scores, and adjusted the results for age and gender. Age, male gender, hypertension, heart failure, undergoing surgery and log first $\mathrm{SCr}$ best predicted preadmission $\mathrm{SCr}$ (adjusted $\mathrm{R}^{2}=0.56$ ) (Table 7). Using log MDRD computed SCr, age, hypertension, and heart failure were still included in the final model, as well as diabetes and SOFA score (Table 7). However, the model did not perform as well as with log first $\mathrm{SCr}$ (adjusted $\mathrm{R}^{2}=0.28$ ).

We then assessed whether there would be improvement in AKI classification with predicted $\mathrm{SCr}$ from multilinear regression models. When using $\mathrm{SCr}$ obtained from the first $\mathrm{SCr}$ model, the AKI incidence increased to $23.9 \%$ (kappa $=0.92 ; 95 \%$ CI 0.89-0.96). When using SCr obtained from the MDRD SCr model, the incidence of AKI remained unchanged (26.7\%; kappa $=0.89 ; 95 \%$ CI 0.84-0.93).

Table 7 Multiple regression analysis to predict preadmission serum creatinine

\begin{tabular}{|c|c|c|}
\hline \multirow[b]{2}{*}{$\begin{array}{l}\text { Covariates or factor included } \\
\text { in model }\end{array}$} & \multicolumn{2}{|c|}{$\begin{array}{l}\text { Dependent Variable } \\
\text { Log (preadmission SCr) }\end{array}$} \\
\hline & Model 1 & Model 2 \\
\hline Constant & 0.844 (0.049) & $-3.728(2.400)$ \\
\hline $\begin{array}{l}\text { Log (first SCr) } \\
\text { Log (MDRD computed SCr) } \\
\text { Age } \\
\text { Male gender }\end{array}$ & $\begin{array}{l}0.461^{\mathrm{a}}(0.026) \\
\mathrm{n} / \mathrm{a} \\
0.001^{\mathrm{b}}(0.000) \\
0.061^{\mathrm{a}}(0.010)\end{array}$ & $\begin{array}{l}\mathrm{n} / \mathrm{a} \\
2.744^{\mathrm{e}}(1.233) \\
0.006^{\mathrm{a}}(0.001) \\
-0.209(0.139)\end{array}$ \\
\hline $\begin{array}{l}\text { Hypertension } \\
\text { COPD }\end{array}$ & $\begin{array}{l}0.029^{c}(0.011) \\
-0.011(0.012)\end{array}$ & $\begin{array}{l}0.048^{\mathrm{a}}(0.014) \\
-0.005(0.015)\end{array}$ \\
\hline Heart failure & $0.037^{d}(0.015)$ & $0.099^{\mathrm{a}}(0.019)$ \\
\hline Diabetes & $0.014(0.011)$ & $0.034^{d}(0.014)$ \\
\hline $\begin{array}{l}\text { Cancer } \\
\text { Surgery } \\
\text { SOFA score } \\
\text { Observations } \\
R^{2} \\
\text { Adjusted } R^{2}\end{array}$ & $\begin{array}{l}0.015(0.011) \\
0.027^{\mathrm{a}}(0.010) \\
-0.001(0.001) \\
498 \\
0.57 \\
0.56\end{array}$ & $\begin{array}{ll}0.020 & (0.014) \\
-0.018 & (0.013) \\
0.006^{\mathrm{b}} & (0.002) \\
498 & \\
0.30 & \\
0.28 & \end{array}$ \\
\hline
\end{tabular}

Regression coefficients for covariates or factor (SEM in parentheses) ${ }^{\mathrm{a}} p \leq 0.001 .{ }^{\mathrm{b}} p=0.002 .{ }^{\mathrm{c}} p=0.01 .{ }^{\mathrm{d}} p=0.02 .{ }^{\mathrm{e}} p=0.03$

$S C r$ serum creatinine, MDRD Modification of Diet in Renal Disease, COPD chronic obstructive pulmonary disease, SOFA Sequential Organ Failure Assessment score

\section{Discussion}

Missing preadmission $\mathrm{SCr}$ values are a common obstacle in AKI research. The use of surrogate methods for baseline $\mathrm{SCr}$ allows researchers to avoid selection bias, but could lead to inaccurate AKI diagnosis and prognosis. In 2004, the Acute Dialysis Quality Initiative (ADQI) group suggested using a surrogate computed $\mathrm{SCr}$ from an eGFR of $75 \mathrm{ml} / \mathrm{min}$ per $1.73 \mathrm{~m}^{2}$ for missing baseline $\mathrm{SCr}$ based on expert opinions [9]. However, it is not entirely clear what is recommended if the first $\mathrm{SCr}$ at hospital admission is lower than the eGFR of $75 \mathrm{ml} / \mathrm{min}$ per $1.73 \mathrm{~m}^{2}$ estimate. Despite an increasing number of AKI studies published over the last decade [26-31], only a few have evaluated the clinical relevance of using different surrogate baseline $\mathrm{SCr}$ to assess AKI diagnosis and outcomes [5, 7, 10-17].

We report our findings using four different surrogate methods (MDRD, CKD-EPI, minimal and first SCr) and imputation strategies to estimate preadmission $\mathrm{SCr}$ for AKI diagnosis. In our study, preadmission $\mathrm{SCr}$ was missing in $50 \%$ of patients, which is similar to previous results from the literature $[5-7,15]$. Patients without preadmission $\mathrm{SCr}$ are less likely to have medical follow-up and may be healthier than those with medical follow-up. As expected, we found that patients without preadmission $\mathrm{SCr}$ were younger and suffered from less comorbidities including CKD (by chart review) than patients with available preadmission $\mathrm{SCr}$.

To our knowledge, our study is the first to simultaneously compare these four methods for estimating baseline $\mathrm{SCr}$ for AKI diagnosis. Our results show that measures to estimate baseline $\mathrm{SCr}$ either underestimate or overestimate AKI incidence, which could affect outcomes associated with presumed AKI. In our population, the AKI incidence was $25.1 \%$ with preadmission $\mathrm{SCr}$ compared to $22.5 \%$ using the first $\mathrm{SCr}, 26.7 \%$ with MDRD, 27.1\% with CKD-EPI, and 43.2\% with the minimal SCr. The first SCr at hospital admission, CKD-EPI and MDRD methods had very good agreement scores with preadmission $\mathrm{SCr}$ to diagnose AKI. The minimal SCr within two weeks after ICU admission had only moderate agreement. Previous studies have also shown that the use of first $\mathrm{SCr}$ can decrease AKI incidence $[11,15]$ and lower sensitivity for AKI diagnosis. These findings have clinical implications since failure to diagnose AKI with lack of preventive and therapeutic measures is different than overdiagnosing AKI and provide unnecessary treatments. Similar to our findings, a few studies have shown that the MDRD method tends to overestimate the incidence of AKI [5, 7, 10, 11, 14, 15]. Two studies have shown that the CKD-EPI overestimates AKI incidence [10, 17]. The magnitude of the overestimation is related to the prevalence of CKD. In our study, the baseline eGFR $\left(89 \mathrm{ml} / \mathrm{min}\right.$ per $\left.1.73 \mathrm{~m}^{2}\right)$ 
was either similar $[10,14,26]$ or higher $[5,11,12]$ than in other studies, which improved the ability of the MDRD and CKD-EPI methods to accurately diagnose AKI. Importantly, we found that the minimal SCr within two weeks after ICU admission markedly overestimated the incidence of AKI and thereby reduced its specificity. $\mathrm{SCr}$ values from the minimal $\mathrm{SCr}$ method were more often lower than the preadmission $\mathrm{SCr}$, possibly due to fluid administration, decreased creatinine production or cessation of renin- angiotensin-aldosterone system blockers $[18,19,32]$. Some studies have assessed the performance of different definitions of minimal SCr on AKI diagnosis with conflicting results $[10,11,16]$. In practice, the use of minimal inpatient SCr for AKI diagnosis is not ideal, as the diagnosis of AKI can only be made retrospectively. Importantly, to our knowledge, the only study that has simultaneously compared first SCr, MDRD and minimal SCr for AKI diagnosis showed comparable trends regarding the overestimation or underestimation of AKI for each method [11].

Regarding AKI staging, the first $\mathrm{SCr}$ at hospital admission provided better accuracy than MDRD computed $\mathrm{SCr}$ in our population, with a misclassified AKI staging rate of $7.4 \%$. The MDRD misclassified $12.2 \%$ of AKI stages, CKD-EPI, 13.1\%, and the minimal SCr, $26.1 \%$. Siew and colleagues also showed that the first SCr performs better for AKI staging than MDRD and minimal SCr [11]. Pickering and colleagues found similar misclassified rate with MDRD SCr than our study (11.1\%) [10]. The rates of misclassification found by Siew and colleagues were higher than in our study [11]. Differences in the prevalence of CKD and timing or severity of AKI severity partly explain these results. A higher proportion of CKD will increase misclassification associated with MDRD SCr and CKD-EPI SCr, while AKI at hospital admission will increase misclassification with the first $\mathrm{SCr}$ method. A higher need for RRT will reduce misclassification with all different surrogate methods, as these will all be categorized as AKI stage 3 .

To our knowledge, our study is the first to assess the performance of four different surrogate methods on the rates of renal recovery after hospital discharge. Assessing renal recovery after hospital discharge avoids systematic bias related to decreases in SCr after critical illness that persist until hospital discharge [33]. Using preadmission SCr, we found that $88.0 \%$ of AKI patients had renal recovery after hospital discharge. The first $\mathrm{SCr}$ was the only surrogate method not associated with a significantly lower performance for renal recovery (89.4\%) compared to preadmission $\mathrm{SCr}$. The minimal, MDRD and CKDEPI computed SCr methods all significantly underestimated renal recovery. As the MDRD and CKD-EPI computed $\mathrm{SCr}$ will underestimate the preadmission $\mathrm{SCr}$ in $C K D$, the rates of renal recovery are expected to be lower with these surrogate methods. More importantly, the percentage of non-renal recovery will be higher as the percentage of CKD patients increases. The MDRD and CKD-EPI surrogate methods should not be used to estimate renal recovery in populations with high prevalence of CKD. The minimal SCr was associated with a lower renal recovery rate possibly due to decreased muscle mass and falsely low SCr values during hospital stay [33]. Our study was underpowered to assess differences in mortality and showed similar percentages of mortality rates for the different surrogate methods. Nevertheless, there were some misclassifications of AKI deaths which could reduce the validity of risk-prediction models for AKI mortality.

It is well known that GFR decreases with age and comorbidities such as long-term diabetes and hypertension and older age [34-36]. In our predictive models with first SCr or MDRD computed SCr, older age and comorbidities like hypertension and heart failure improved the prediction of baseline SCr. Being male or suffering from diabetes also helped to predict baseline $\mathrm{SCr}$ with first $\mathrm{SCr}$ or MDRD computed SCr, respectively. These results were expected as these variables are known to be associated with a higher likelihood of CKD. We also found that a higher SOFA scores helped to predict baseline SCr from MDRD computed SCr, and being admitted for surgery helped to predict baseline SCr from the first SCr. These results may reflect underlying comorbidities associated with these findings. Imputation methods minimally increased the agreement for AKI diagnosis.

Our study has several strengths. We included critically ill patients from specialized intensive care units (cardiac surgery, neurosurgery/neurological, trauma, medical) with detailed data on patient characteristics and had limited exclusion criteria to increase generalizability. We assessed the performance of four commonly used surrogate methods not only for AKI diagnosis and staging, but also for renal recovery and mortality. Our study also has limitations. The pattern of missing preadmission $\mathrm{SCr}$ and percentage of CKD in our academic center may not be similar in other centers, and baseline kidney function may be different between patients with and without baseline creatinine, affecting AKI diagnosis by estimating methods. However, we found a similar percentage of missing baseline SCr [12] and comparable baseline eGFR [10, 14, 29] in other large epidemiological AKI studies. Also, almost all patients were Caucasian or Asian. Therefore, our results may not be applicable to patients from other races. This study was performed in critically ill patients and results may be different in elective surgical patients or those admitted to hospital wards. We chose to define baseline $\mathrm{SCr}$ as the closest value between 3 and 12 months before admission to reflect CKD diagnosis by the KDIGO [24], and if unavailable, the value furthest to 90 days before admission. Other studies have chosen SCr values 7-365 
days before admission which may affect overall results $[15,37]$. Finally, with a larger database, we might have shown statistically different mortality rates between surrogate methods and preadmission $\mathrm{SCr}$ in AKI patients.

\section{Conclusion}

Our results have important research and clinical implications. The use of different surrogate methods can significantly affect AKI incidence and outcomes. In our cohort, the first $\mathrm{SCr}$ had a better agreement for diagnosing and staging AKI than MDRD, CKD-EPI and minimal SCr. Our results concur with the recommendation from the European Renal Best Practice position statement on the KDIGO guidelines to use the first $\mathrm{SCr}$ of the episode when baseline $\mathrm{SCr}$ is missing [38]. However, MDRD SCr and CKD-EPI improved AKI diagnosis sensitivity compared to first $\mathrm{SCr}$ in our population having a relatively low prevalence of CKD (16\%). Importantly, even with a low prevalence of CKD, MDRD and CKD-EPI SCr significantly underestimated renal recovery after hospital discharge compared to other methods. Therefore, research using MDRD or CKD-EPI computed $\mathrm{SCr}$ as surrogate methods for missing baseline $\mathrm{SCr}$ should be interpreted with caution in populations with a higher prevalence of CKD. Similarly, the use of minimal SCr during ICU stay also underestimates renal recovery after hospital discharge. Our multilinear regression model identified several variables helping to predict baseline SCr. However, imputation methods only minimally increased agreement for AKI diagnosis. Further research is needed on the effect of using surrogate methods and imputation methods for baseline SCr on AKI diagnosis and outcomes.

\section{Additional files}

Additional file 1: Figure S1a-1d. Bland-Altman methods between preadmission serum creatinine and various surrogates methods for estimating baseline serum creatinine. (a) first serum creatinine, (b) minimal serum creatinine, (c) MDRD computed serum creatinine, (d) CKD-EPI computed serum creatinine. SCr: serum creatinine; MDRD: Modification of Diet in Renal Disease; CKD-EPI: Chronic Kidney Disease Epidemiology Collaboration. (PNG 78 kb)

Additional file 2: Table S1a. Comparison between well-classified and misclassified acute kidney injury by the first serum creatinine method. Table S1b. Comparison between well-classified and misclassified acute kidney injury by the Modification of Diet in Renal Disease (MDRD) method. Table S1c. Comparison between well-classified, missed and overdiagnosed acute kidney injury by the first serum creatinine method. Table S1d. Comparison between well-classified, missed and overdiagnosed acute kidney injury by the Modification of Diet in Renal Disease (MDRD) method. (DOCX $87 \mathrm{~kb}$ )

\section{Abbreviations}

AKI: acute kidney injury; AQQI: Acute dialysis quality initiative; CAD: coronary artery disease; CKD: chronic kidney disease; CKD-EPI: Chronic kidney disease epidemiology collaboration; COPD: chronic obstructive pulmonary disease; eGFR: estimated glomerular filtration rate; ICU: intensive care unit;
IQR: interquartile range; KDIGO: Kidney disease improving global outcomes; LOS: lengths of stay; MDRD: Modification of diet in renal disease; RRT: renal replacement therapy; SCr: serum creatinine; SOFA: Sequential organ failure assessment; STROBE: STrengthening the reporting of observational studies in epidemiology

\section{Acknowledgements}

We would like to thank Mr. Thierry Ducruet for statistical assistance. Some of the results of this study have been presented at the 2015 American Society of Nephrology meeting.

\section{Funding}

J.B and S.T.'s research efforts are supported by the Fonds de la Recherche du Québec-Santé. This study was supported by a grant from the Kidney Foundation of Canada. These funding agencies did not have any role in the design of the study and collection, analysis, and interpretation of data and in writing the manuscript.

\section{Availability of data and materials}

The datasets generated during and/or analysed during the current study are not publicly available because of a restriction from our ethics' committee but are available from the corresponding author on request if approved by our ethics' committee.

\section{Authors' contributions}

Co-authors have all contributed to this manuscript and approve its submission. Conception or design (ABJ, WBS, RG, FM, FP, ST) or analysis and interpretation of data, or both (JB). Drafting the article (ABJ, WBS, JB) or revising it (RG, FM, FP, $\mathrm{ST}, \mathrm{JB})$. Providing intellectual content of critical importance to the work described (ABJ, WBS, RG, FM, FP, ST, JB). Final approval of the version to be published (ABJ, WBS, RG, FM, FP, ST, JB). All authors read and approved the final manuscript.

\section{Competing interests}

All authors declare that they have no competing interests.

\section{Consent for publication}

Not applicable.

\section{Ethics approval and consent to participate}

The Sacre-Coeur Hospital ethics' committee approved the study. Written consent was waived because of the retrospective observational nature of the study.

\section{Publisher's Note}

Springer Nature remains neutral with regard to jurisdictional claims in published maps and institutional affiliations.

Received: 21 January 2016 Accepted: 16 April 2017

Published online: 28 April 2017

\section{References}

1. Chertow GM, Burdick E, Honour M, Bonventre JV, Bates DW. Acute kidney injury, mortality, length of stay, and costs in hospitalized patients. J Am Soc Nephrol. 2005;16(11):3365-70.

2. Waikar SS, Liu KD, Chertow GM. Diagnosis, epidemiology and outcomes of acute kidney injury. Clin J Am Soc Nephrol. 2008;3(3):844-61.

3. Chawla LS, Amdur RL, Amodeo S, Kimmel PL, Palant CE. The severity of acute kidney injury predicts progression to chronic kidney disease. Kidney Int. 2011;79(12):1361-9.

4. Kidney Disease: Improving Global Outcomes (KDIGO) Acute Kidney Injury Work Group. KDIGO Clinical Practice Guideline for Acute Kidney Injury. Kidney Int Suppl. 2012;2:1-138.

5. Bagshaw SM, Uchino S, Cruz D, Bellomo R, Morimatsu H, Morgera S, Schetz M, Tan I, Bouman C, Macedo E, et al. A comparison of observed versus estimated baseline creatinine for determination of RIFLE class in patients with acute kidney injury. Nephrol Dial Transplant. 2009;24(9):2739-44.

6. Hoste EA, Clermont G, Kersten A, Venkataraman R, Angus DC, De Bacquer D, Kellum JA. RIFLE criteria for acute kidney injury are associated with hospital mortality in critically ill patients: a cohort analysis. Crit Care. 2006;10(3):R73. 
7. Sims AJ, Hussein HK, Prabhu M, Kanagasundaram NS. Are surrogate assumptions and use of diuretics associated with diagnosis and staging of acute kidney injury after cardiac surgery? Clin J Am Soc Nephrol. 2012;7(1):15-23

8. Siew ED, Matheny ME. Choice of Reference Serum Creatinine in Defining Acute Kidney Injury. Nephron. 2015;131(2):107-12.

9. Bellomo R, Ronco C, Kellum JA, Mehta RL, Palevsky P. Acute renal failure definition, outcome measures, animal models, fluid therapy and information technology needs: the Second International Consensus Conference of the Acute Dialysis Quality Initiative (ADQI) Group. Crit Care. 2004;8(4):R204-212.

10. Pickering JW, Endre ZH. Back-calculating baseline creatinine with MDRD misclassifies acute kidney injury in the intensive care unit. Clin J Am Soc Nephrol. 2010;5(7):1165-73.

11. Siew ED, Matheny ME, Ikizler TA, Lewis JB, Miller RA, Waitman LR, Go AS, Parikh CR, Peterson JF. Commonly used surrogates for baseline rena function affect the classification and prognosis of acute kidney injury. Kidney Int. 2010;77(6):536-42.

12. Siew ED, Peterson JF, Eden SK, Moons KG, Ikizler TA, Matheny ME. Use of multiple imputation method to improve estimation of missing baseline serum creatinine in acute kidney injury research. Clin J Am Soc Nephrol. 2013;8(1):10-8.

13. Zappitelli M, Parikh CR, Akcan-Arikan A, Washburn KK, Moffett BS, Goldstein SL. Ascertainment and epidemiology of acute kidney injury varies with definition interpretation. Clin J Am Soc Nephrol. 2008;3(4):948-54.

14. Zavada J, Hoste E, Cartin-Ceba R, Calzavacca P, Gajic O, Clermont G, Bellomo $R$, Kellum JA, investigators AKI. A comparison of three methods to estimate baseline creatinine for RIFLE classification. Nephrol Dial Transplant. 2010;25(12):3911-8.

15. Thongprayoon C, Cheungpasitporn W, Harrison AM, Kittanamongkolchai W, Ungprasert P, Srivali N, Akhoundi A, Kashani KB. The comparison of the commonly used surrogates for baseline renal function in acute kidney injury diagnosis and staging. BMC Nephrol. 2016;17:6.

16. Thongprayoon C, Cheungpasitporn W, Kittanamongkolchai W, Srivali N, Ungprasert P, Kashani K. Optimum methodology for estimating baseline serum creatinine for the acute kidney injury classification. Nephrology. 2015;20(12):881-6.

17. Bernardi MH, Schmidlin D, Ristl R, Heitzinger C, Schiferer A, Neugebauer T, Wrba T, Hiesmayr M, Druml W, Lassnigg A. Serum Creatinine Back-Estimation in Cardiac Surgery Patients: Misclassification of AKI Using Existing Formulae and a Data-Driven Model. Clin J Am Soc Nephrol. 2016;11(3):395-404.

18. Macedo E, Bouchard J, Soroko SH, Chertow GM, Himmelfarb J, Ikizler TA Paganini EP, Mehta RL. Program to Improve Care in Acute Renal Disease S: Fluid accumulation, recognition and staging of acute kidney injury in critically-ill patients. Crit Care. 2010;14(3):R82.

19. Prowle JR, Leitch A, Kirwan CJ, Forni LG. Positive fluid balance and AK diagnosis: assessing the extent and duration of 'creatinine dilution'. Intensive Care Med. 2015;41(1):160-1.

20. Schetz M, Gunst J, Van den Berghe G. The impact of using estimated GFR versus creatinine clearance on the evaluation of recovery from acute kidney injury in the ICU. Intensive Care Med. 2014;40(11):1709-17.

21. Levey AS, Bosch JP, Lewis JB, Greene T, Rogers N, Roth D. A more accurate method to estimate glomerular filtration rate from serum creatinine: a new prediction equation. Modification of Diet in Renal Disease Study Group. Ann Intern Med. 1999:130(6):461-70.

22. Levey AS, Stevens LA, Schmid CH, Zhang YL, Castro 3rd AF, Feldman HI, Kusek JW, Eggers $P$, Van Lente $F$, Greene $T$, et al. A new equation to estimate glomerular filtration rate. Ann Intern Med. 2009;150(9):604-12.

23. von Elm E, Altman DG, Egger M, Pocock SJ, Gotzsche PC, Vandenbroucke JP, Initiative S. The Strengthening the Reporting of Observational Studies in Epidemiology (STROBE) statement: guidelines for reporting observational studies. Ann Intern Med. 2007;147(8):573-7.

24. Stevens PE, Levin A. Kidney Disease: Improving Global Outcomes Chronic Kidney Disease Guideline Development Work Group M: Evaluation and management of chronic kidney disease: synopsis of the kidney disease: improving global outcomes 2012 clinical practice guideline. Ann Intern Med. 2013;158(11):825-30.

25. Vincent JL, Moreno R, Takala J, Willatts S, De Mendonca A, Bruining H, Reinhart CK, Suter PM, Thijs LG. The SOFA (Sepsis-related Organ Failure Assessment) score to describe organ dysfunction/failure. On behalf of the Working Group on Sepsis-Related Problems of the European Society of Intensive Care Medicine. Intensive Care Med. 1996:22(7):707-10.
26. Hoste EA, Bagshaw SM, Bellomo R, Cely CM, Colman R, Cruz DN, Edipidis K, Forni LG, Gomersall CD, Govil D, et al. Epidemiology of acute kidney injury in critically ill patients: the multinational AKI-EPI study. Intensive Care Med. 2015:41(8):1411-23.

27. Grams ME, Sang Y, Coresh J, Ballew S, Matsushita K, Molnar MZ, Szabo Z, Kalantar-Zadeh K, Kovesdy CP. Acute Kidney Injury After Major Surgery: A Retrospective Analysis of Veterans Health Administration Data. Am J Kidney Dis. 2016; 67(6):872-80.

28. Lin J, Fernandez H, Shashaty MG, Negoianu D, Testani JM, Berns JS, Parikh CR, Wilson FP. False-Positive Rate of AKI Using Consensus Creatinine-Based Criteria. Clin J Am Soc Nephrol. 2015;10(10):1723-31.

29. Bouchard J, Acharya A, Cerda J, Maccariello ER, Madarasu RC, Tolwani AJ, Liang X, Fu P, Liu ZH, Mehta RL. A Prospective International Multicenter Study of AKI in the Intensive Care Unit. Clin J Am Soc Nephrol. 2015;10(8): 1324-31.

30. Siew ED, Parr SK, Abdel-Kader K, Eden SK, Peterson JF, Bansal N, Hung AM, Fly J, Speroff T, Ikizler TA et al. Predictors of Recurrent AKI. J Am Soc Nephrol. 2016; 27(4):1190-200

31. Renoux C, Lix LM, Patenaude V, Bresee LC, Paterson JM, Lafrance JP, Tamim H, Mahmud SM, Alsabbagh MW, Hemmelgarn B, et al. Serotonin-Norepinephrine Reuptake Inhibitors and the Risk of AKl: A Cohort Study of Eight Administrative Databases and Meta-Analysis. Clin J Am Soc Nephrol. 2015;10(10):1716-22.

32. Doi K, Yuen PS, Eisner C, Hu X, Leelahavanichkul A, Schnermann J, Star RA. Reduced production of creatinine limits its use as marker of kidney injury in sepsis. J Am Soc Nephrol. 2009;20(6):1217-21.

33. Prowle JR, Kolic I, Purdell-Lewis J, Taylor R, Pearse RM, Kirwan CJ. Serum creatinine changes associated with critical illness and detection of persistent renal dysfunction after AKI. Clin J Am Soc Nephrol. 2014;9(6):1015-23.

34. Berg UB. Differences in decline in GFR with age between males and females. Reference data on clearances of inulin and PAH in potential kidney donors. Nephrol Dial Transplant. 2006;21(9):2577-82.

35. Wetzels JF, Kiemeney LA, Swinkels DW, Willems HL, den Heijer M. Age- and gender-specific reference values of estimated GFR in Caucasians: the Nijmegen Biomedical Study. Kidney Int. 2007;72(5):632-7.

36. Cirillo M, Anastasio P, De Santo NG. Relationship of gender, age, and body mass index to errors in predicted kidney function. Nephrol Dial Transplant. 2005:20(9):1791-8

37. Siew ED, Ikizler TA, Matheny ME, Shi Y, Schildcrout JS, Danciu I, Dwyer JP, Srichai M, Hung AM, Smith JP, et al. Estimating baseline kidney function in hospitalized patients with impaired kidney function. Clin J Am Soc Nephrol. 2012;7(5):712-9.

38. Fliser D, Laville M, Covic A, Fouque D, Vanholder R, Juillard L, Van Biesen W. A European Renal Best Practice (ERBP) position statement on the Kidney Disease Improving Global Outcomes (KDIGO) clinical practice quidelines on acute kidney injury: part 1: definitions, conservative management and contrast-induced nephropathy. Nephrol Dial Transplant. 2012;27(12):4263-72.

\section{Submit your next manuscript to BioMed Central and we will help you at every step:}

- We accept pre-submission inquiries

- Our selector tool helps you to find the most relevant journal

- We provide round the clock customer support

- Convenient online submission

- Thorough peer review

- Inclusion in PubMed and all major indexing services

- Maximum visibility for your research

Submit your manuscript at www.biomedcentral.com/submit 